\title{
Phlebologische Fortbildung für Dermatologen
}

\section{Phlebology and Dermatology}

Autor

Institut

\section{Stücker}

Klinik für Dermatologie, Venenzentrum der Dermatologischen und Gefäßchirurgischen Kliniken, Kliniken der Ruhr-Universität Bochum

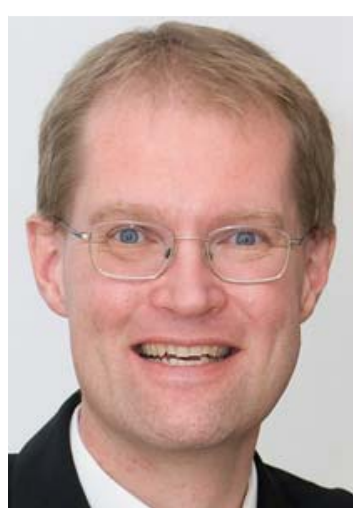

Prof. Dr. med. M. Stücker
Bibliografie

DOI http://dx.doi.org/

10.1055/s-0034-1377957

Akt Dermatol 2014; 40: 479-480

(c) Georg Thieme Verlag KG

Stuttgart · New York

ISSN 0340-2541

Korrespondenzadresse

Prof. Dr. med. Markus Stücker Klinik für Dermatologie

Venenzentrum der Dermatologischen und Gefäßchirurgischen Kliniken Ruhr-Universität Bochum im St. Maria-Hilf-Krankenhaus Hiltroper Landwehr 11-13 44805 Bochum

m.stuecker@

klinikum-bochum.de
Die Phlebologie ist integraler Bestandteil der Dermatologie. In der Weiterbildung zum Facharzt für Haut- und Geschlechtskrankheiten nimmt sie einen ganz wesentlichen Anteil ein, da von den insgesamt 19 verschiedenen Untersuchungs- und Behandlungsverfahren, für die Richtzahlen nachgewiesen werden müssen, vier dem phlebologischen Bereich zugeordnet sind (phlebologischoperative Eingriffe, Sklerosierungstherapie, Doppler-/Duplex-Sonografie peripherer Gefäße, phlebologische Funktionsuntersuchungen wie die Verschlussplethysmografie und Lichtreflexrheografie). Dem wird dadurch Rechnung getragen, dass ein überwiegender Anteil der deutschen Hautkliniken phlebologische Therapien durchführt [1]. Dabei wird der gesamte Bereich der Phlebologie durch Dermatologen abgedeckt. Dies zeigt sich in dem breiten Spektrum der in diesem Schwerpunktheft der Aktuellen Dermatologie zusammengestellten Artikel, die ausnahmslos von Dermatologen verfasst worden sind. Dies gilt für die unterschiedlichen Behandlungstechniken wie die Verödungsbehandlung, thermische Ablationsverfahren mit Kathetern, Varizenoperationen, Behandlung der tiefen Beinvenenthrombose und Vaskulopathien über allergologische und mikrobiologische Fragestellungen der Phlebologie, mikrobiologische Untersuchung bei chronischen Wunden und die sozialökonomische Bedeutung chronischer Wunden bis hin zu den Krankheitsbildern des Lymphödems und des Lipödems.

Die Phlebologie hat in den letzten Jahren eine rasante Entwicklung durchgemacht. Dies hat zu einer wesentlich verbesserten phlebologischen Versorgung der Bevölkerung in Deutschland geführt. Hierdurch konnten die schweren Stadien der chronischen Veneninsuffizienz dramatisch reduziert werden. So sind die klinischen Stadien C4 bis C6 (Patienten mit Hautkomplikationen vom Ekzem bis hin zum Ulcus cruris venosum) in ihrer Häufigkeit dramatisch gesunken: Von 13\% der Bevölkerung in Deutschland in der Tübinger Studie 1981 auf 3,3\% in der Bonner Venenstudie [2]. Diese Weiterentwicklung der phlebologischen Therapie zeigt sich zum Beispiel in der individualisierten Therapie der Stammveneninsuffizienz, wie wir sie heute ausüben können. Statt schematisch eine Strippingoperation von der Leiste bis zum Knöchel nach der Technik von Babcock durchzuführen, gibt es heute neben der minimal-invasiv durchführbaren Crossektomie und Saphenektomie die Möglichkeit einer thermischen oder chemischen endoluminalen Ablation, der Venenklappenrekonstruktion durch extraluminale Valvuloplastien und auch die Operationstechniken nach CHIVA. Aufgrund der positiven Entwicklung der Phlebologie mit neuen diagnostischen und therapeutischen Möglichkeiten ist eine kontinuierliche Fortbildung für Dermatologen in diesem Bereich sinnvoll. Daher bin ich den Herausgebern der Aktuellen Dermatologie außerordentlich dankbar für die Möglichkeit dieses Schwerpunkthefts.

Viele niedergelassene Kollegen konnten während ihrer Facharztausbildung die Zusatzbezeichnung Phlebologie nicht erwerben und können damit auch nur eigeschränkt nach außen ihre oftmals vorhandene phlebologische Expertise dokumentieren. Gleichzeitig ist es oft schwer zu belegen, dass der Inhaber der Zusatzbezeichnung immer noch auf dem aktuellen Wissensstand ist. Aufgrund dieser Problematik hat die Deutsche Gesellschaft für Phlebologie, die traditionell zu einem wesentlichen Anteil von Dermatologen getragen wird, die Fortbildungsakademie der DGP gegründet, welche ein phlebologisches Fortbildungszertifikat anbietet. Unabhängig vom Erwerb der Zusatzbezeichnung Phlebologie kann hier eine kontinuierliche Fortbildung im phlebologischen Bereich dokumentiert werden. Dieses phlebologische Fortbildungszertifikat kann von allen DGP-Mitgliedern durch eine systematische Teilnahme an offiziellen Fortbildungsaktivitäten 
der DGP oder von der DGP offiziell anerkannten Fortbildungsveranstaltungen mit mindestens 20 Fortbildungspunkten im phlebologischen Bereich pro Jahr erworben werden. In systematischer Form werden verschiedene Formate angeboten. Genannt seien:

- Basiskurs Phlebologie über 2 Tage

- Crashkurs Phlebologie über 1/2 Tag (Auffrischung des phlebologischen Basiswissens)

- Update Phlebologie über $1 \frac{1}{2}$ Tag (Übersicht über die aktuellsten phlebologischen Publikationen der letzten 2 Jahre)

- Hands-On-Kurse (Kurse mit praktischem Schwerpunkt zum Erlernen neuer Techniken wie Schaumsklerosierung, Laserund Radiofrequenz-Ablation, Duplexsonografie)

Welchen Stellenwert hat die Phlebologie nun für die Hautkliniken und die Hautarztpraxen? Für die Kliniken ist die kompetente Behandlung chronischer Wunden wie des Ulcus cruris venosum heute essentiell. Kaum eine dermatologische Indikation gehört so sicher in das stationäre Behandlunsspektrum wie die chronische Wunde. Die komplexe Behandlung dieser oft schwerkranken Patienten hat eine entsprechend hohe Bewertungsrelation im DRG-System. Das gesamte Spektrum der Phlebologie von der Besenreiserbehandlung über die Therapie der Varikosis und der tiefen Beinvenenthrombose bis hin zur Diagnostik der chronischen Wunden ist aus der dermatologischen Praxis nicht wegzudenken. Hier bietet die Plebologie ein großes Betätigungsfeld, wenn man die Zahlen der Bonner Venenstudie aus dem Jahr 2003 berücksichtigt: 6,9\% der Bevölkerung werden nach diesen Daten an den Venen operiert, 5,5\% verödet, 14,6\% tragen Kompressionsstrümpfe.

Vor diesem Hintergrund werden sicherlich viele Kolleginnen und Kollegen in diesem Schwerpunktheft nützliche Aspekte für die tägliche Versorgung unserer Patienten finden.

\section{Literatur}

1 Reich-Schupke S, Alm J, Altmeyer P et al. Phlebologie an deutschen Hautkliniken. Eine Bestandsaufnahme im Auftrag der Deutschen Gesellschaft für Phlebologie. Hautarzt 2013; 64: 685-694

2 Rabe E, Pannier-Fischer $F$, Bromen $K$ et al. Bonner Venenstudie der Deutschen Gesellschaft für Phlebologie. Epidemiologische Untersuchung zur Frage der Häufigkeit und Ausprägung von chronischen Venenkrankheiten in der städtischen und ländlichen Wohnbevölkerung. Phlebologie 2003; 32: 1-14 